\title{
Competencia para el diseño y la ejecución del proceso de enseñanza-aprendizaje en la Facultad de Ciencias Administrativas y Económicas, Universidad Técnica del Norte de Ecuador
}

\author{
Omar Abreu-Valdivia, Soraya Rhea-González, Gabriela Arciniegas-Romero y Sandra Guevara-López \\ Universidad Técnica del Norte, Campus. Avenida 17 de julio, 5-21, General José María Córdova, Código postal 100105, \\ Ibarra, Ecuador (correo-e: oabreu@utn.edu.ec, srheag@utn.edu.ec,mgarciniegas@utn.edu.ec,
}

smguevaral@utn.edu.ec).

Recibido Ene. 7, 2020; Aceptado Mar. 11, 2020; Versión final Abr. 1, 2020, Publicado Ago. 2020

\begin{abstract}
Resumen
En este artículo se presentan los resultados del sistema de capacitación para el desarrollo de la competencia para el diseño y la ejecución del proceso de enseñanza-aprendizaje en los docentes de la Facultad de Ciencias Administrativas y Económicas (FACAE) de la Universidad Técnica del Norte (UTN) de Ecuador. En la investigación se utilizaron métodos, técnicas e instrumentos como el Kuder Richardson- 20, el Alfa de Cronbach, el histórico, la observación, la entrevista, la encuesta, la revisión documental, el estadístico, el análisis, la síntesis, el lógico, el sistémico y el hermenéutico. Entre los resultados destacan la sistematización de referentes relacionados con la competencia para el diseño y la ejecución del proceso de enseñanzaaprendizaje y la demostración de que la competencia para el diseño y la ejecución del proceso de enseñanzaaprendizaje está asociada directamente a las fases más importantes de la clase: la preparación previa, la introducción, el desarrollo y las conclusiones.
\end{abstract}

\section{Competence for the design and execution of the teaching- learning process at the Faculty of Administrative and Economic Sciences, Technical University of Northern Ecuador}

\begin{abstract}
This article presents the results of the training system in the development of the competence for the design and execution of the teaching-learning process in the teachers of the Faculty of Administrative and Economic Sciences of the North Technical University (UTN) from Ecuador. The research methods include the following: Kuder Richardson-20, Cronbach's Alpha, historical data, observational data, interviews, surveys, documentary reviews, statistical analyses, synthesis, logical, and systemic and hermeneutic tools. Result highlights include the systematization of references related to competence for the design and execution of the teaching-learning process and demonstration that the competence for the design and execution of the teaching-learning process is associated directly to the most import phases of the class: preparation, introduction, development, and conclusions.
\end{abstract}




\section{INTRODUCCIÓN}

La formación docente es una tarea de máxima complejidad. Requiere tiempo, dedicación, disciplina consciente, interés, disposición, recursos y políticas bien concebidas. Esa complejidad es mucho mayor en tiempos de la sociedad del conocimiento y de la globalización de la ciencia y la tecnología. Se trata de establecer un equilibro entre diversos componentes como el sistema teórico y práctico de ciencias como la Pedagogía (Ramos et al., 2017) la Didáctica (Abreu et al., 2018) y disciplinas como la metodología y de diferenciar el objeto de estudio y las funciones de cada una (Abreu et al., 2017). En la FACAE de la UTN de Ecuador se trabaja en la formación de los docentes que la integran, para desarrollar las competencias que garanticen un ejercicio académico de calidad. La facultad acoge a las carreras de Administración de empresas, Contabilidad y auditoría, Contaduría pública y auditoría, Economía mención finanzas, Mercadotecnia, Turismo, Gastronomía y Derecho. El reto es exigente si se tiene en cuenta que más del 90 \% sus profesores no ha recibió ese tipo de formación previa a su desempeño profesional. Esta situación tiene una influencia directa en la formación integral de los estudiantes. Por tales razones, se desarrolla el proyecto de investigación "Sistema de capacitación para la relación contenidos, métodos, evaluación, aprendizaje desarrollador para los docentes de la FACAE", parte de los esfuerzos para la formación continua de profesionales necesitados del dominio de estos contenidos (Abreu et al., 2016).

El concepto de competencia ha sido objeto de análisis de números autores. Las competencias se corresponden con un modo de actuación idóneo respecto a una tarea concreta y contextualizada. Es un término asimilado con propiedad, aplicado de manera flexible en una situación determinada, para encontrar soluciones variadas y pertinentes. En las competencias convergen comportamientos sociales, afectivos, cognoscitivos, psicológicos y sensoriales, los cuales influyen en el desempeño de una función y en el desarrollo de una actividad de carácter dificultoso y múltiples complicaciones. Las competencias laborarles se han convertido en un elemento determinante en la selección de un candidato para que desempeñe alguna labor dentro de una organización. Un ser humano competente está más cerca del éxito que aquel que no lo es. Estas se refieren a conocimientos, habilidades, capacidades, actitudes, aptitudes, valores y disposición de una persona para cumplir eficientemente determinada actividad en medio de situaciones que no tradicionales.

Actualmente las competencias son cada vez más valoradas por los responsables de los organismos empleadores. Su desarrollo hace al ser humano apto para enfrentar los desafíos laborales. Esta condición garantiza el desenvolvimiento de una persona en la actividad que desempeña y determina la calidad del resultado de la misma. Bien logradas, establecen diferencias competitivas entre los profesionales, contribuyen a la caracterización objetiva de cada uno, deciden su selección en el mercado laboral y caracterizan su trabajo. En esencia, las competencias son resultado de procesos complejos. Se desarrollan en la formación continua y se derivan de la metacognicón. Están asociadas al desempeño en la ejecución de responsabilidades. Requieren una actuación idónea en ese desempeño. Son manifestaciones individuales, pero facilitan la colaboración, el trabajo en equipo y la integración social. Son útiles para la solución de problemas contextualizados. Distinguen al ser humano y son altamente valoradas por la comunidad. Evidencian la madurez y las potencialidades de la personalidad para el proceso de enseñanza-aprendizaje. Precisan del vínculo de la teoría con la práctica. Exigen la complementación de intereses individuales, organizacionales y sociales. Integran saberes, habilidades, capacidades, conductas, valores y demostraciones. Tienen un componente ético relevante y son altamente estimadas en la sociedad del conocimiento.

Los desafíos del siglo XXI imponen la actualización sistemática de las competencias profesionales. Estas deben asumirse como necesidades que equipan a las personas, con herramientas, habilidades, nuevas formas de pensar, trabajar y vivir, para la vida contemporánea y futura (Caena y Redecker, 2019). El estudio de la teoría acerca de competencias revela numerosas clasificaciones. La mayoría de estas pone el acento en dos tipos de competencias, una denominada de desarrollo técnico-profesional y otra de desarrollo socio-personal. La primera, también denominada funcionales, se circunscribe alrededor de conocimientos y procedimientos para un ámbito profesional o especialización. Se vincula con el "saber" y el "saber hacer", aspectos necesarios para el desempeño experto en una actividad laboral. Guerrero y De los Ríos (2012) plantean que las investigaciones acerca de competencias profesionales han ganado un protagonismo tan significativo que los investigadores ya elaboran modelos para su desarrollo. Las competencias profesionales devienen eje del modelo que permite trasladarse desde el desempeño hasta el proceso de formación. Se asume el término eje como el aspecto esencial para generar movimiento o el fundamento de un razonamiento o comportamiento determinado, atravesando todo el currículo de formación y expresándose en la integración de contenidos (conocimientos, habilidades normas y valores profesionales). Expresan la concepción curricular que permite estructurar los planes y programas de preparación y tienen la peculiaridad de ser síntesis de todos los fundamentos teóricos del currículo. Son expresiones didácticas de las cualidades del sujeto, en que se sintetizan el ser, el saber, el hacer y el desarrollo de sus capacidades y aptitudes para los procesos profesionales. 
Las competencias docentes no pueden evolucionar al margen de la vida humana. Como responsables de sistemas educativos estos profesionales necesitan priorizar este aspecto formativo, en correspondencia con reformas de este sector, de su propia formación, de los resultados de la ciencia y de otros campos de la sociedad (Selvi, 2010). Los docentes deben repensar su rol en el entorno virtual del proceso de enseñanzaaprendizaje. Tendrán que facilitar situaciones comunicativas adaptadas a varias interrelaciones complejas, profesor alumno, estudiantes, contenidos y aprendizaje asincrónico, lo cual requiere de competencias actualizadas (Guasch et al., 2010). Mohamadi y Malekshahi (2018) dicen que las competencias docentes se definen por tres demisiones. La primera es cognitiva, la segunda es funcional, se refiere a habilidades y actitudes y la tercera, compuesta por actitudes y valores, se relaciona con la autonomía y la responsabilidad del maestro. Estefanova et al., (2019) reconocen la necesidad creciente de identificar el marco de las competencias docentes. Plantean que estas son imprescindibles para desarrollar conocimientos, habilidades y actitudes, propias del aprendizaje durante la vida. Este aspecto ha sido tratado por numerosos autores desde diferentes perspectivas y escenarios. Todos coinciden en la necesidad de un docente competente como garantía de la formación integral de los estudiantes.

McCabe y O'Connor (2014) hablan de la relación entre el aprendizaje de los estudiantes y la responsabilidad del profesor. Villarroel y Bruna (2016) destacan que las competencias pedagógicas deben caracterizar a un docente universitario de excelencia. Gokalp (2016) hace referencia a la necesidad de investigar las competencias de enseñanza en maestros. Barreto da Cruz y Andrade (2017) establecen la relación entre la Didáctica, el desempeño docente y el resultado formativo de los estudiantes. Santos y Sarceda (2017) plantean el desarrollo de competencias docentes en educación infantil. Nousiainen et al., (2018) hablan de competencias docentes en Pedagogía basada en juegos. Vargas y Apablaza (2019) estudian la competencia comunicativa en la formación inicial del profesor de matemática. Las competencias docentes existen en función de la formación integral y contextualizada de los estudiantes. Son resultado de la formación continua del profesional de la educación. Se expresan en el domino profundo del contenido de las materias que imparte, de la Pedagogía, la Didáctica, la Psicología educativa, la metodología y otras ciencias y disciplinas. En la integración al debate profesional. En la vocación por la actualización constante y sistemática. En el conocimiento de las características de sus estudiantes y en la capacidad de utilizarlo para potenciar la formación de estos. En una conducta acorde con valores autóctonos y universales, que sirva de referencia para sus discípulos. En el sentido de la ejemplaridad integradora. En la capacidad para diseñar, conducir, ejecutar y evaluar la formación de sus alumnos y la suya propia. En el modo de actuación para el desarrollo del trabajo académico, la investigación y la vinculación con la comunidad.

La investigación demuestra que, para relacionar contenidos, métodos, evaluación y aprendizaje desarrollador, los docentes deben haber logrado la competencia para el diseño y la ejecución del proceso de enseñanzaaprendizaje y evidenciarlo en su modo de actuación profesional, espacialmente en la clase. Para ello, se incorporó este aspecto al sistema de capacitación. El objetivo de este artículo es explicar los resultados del sistema de capacitación para el desarrollo de la competencia para el diseño y la ejecución del proceso de enseñanza-aprendizaje en la Facultad de Ciencias Administrativas y Económicas de la Universidad Técnica del Norte de Ecuador.

\section{METODOLOGÍA}

La FACAE cuenta con 133 docentes y 2988 estudiantes, en 8 carreras. Del total de docentes 124 participaron en la investigación. Para conocer la satisfacción de los estudiantes con las clases que reciben, antes y después de la capacitación, se les aplicó un cuestionario a 1106, el $37 \%$ de la matrícula, seleccionados mediante un muestreo representativo de tipo aleatorio simple. En la investigación se utilizó el método histórico para el estudio de los referentes relacionados con la competencia para el diseño y la ejecución del proceso enseñanzaaprendizaje. La observación del modo de actuación profesional de los profesores para conocer el desarrollo de dicha competencia en ellos, la relación que establecían entre el componente académico, el investigativo y la vinculación con la comunidad y para determinar potencialidades y debilidades. La entrevista a directivos para conocer su criterio acerca de la manifestación de esta competencia en sus subordinados y la encuesta para determinar los niveles de la mencionada competencia en los educadores. Kuder Richardson- 20 fue útil para calcular la confiabilidad del instrumento aplicado a 15 expertos, cuyas alternativas oscilaban entre el 1 y el 5 , en una escala ascendente. El objetivo fue recopilar el criterio de estos profesionales acerca de la utilidad y el carácter objetivo del instrumento elaborado por los investigadores para evaluar la competencia para el diseño y la ejecución del proceso de enseñanza-aprendizaje en los docentes de la FACAE. Para ello se aplicó la formula (1), el 0.886 que resultó del cálculo demuestra que el instrumento es confiable.

$$
r t t=\frac{n}{n-1} * \frac{v t-\sum p q}{v t}=\frac{40}{40-1} * \frac{28.367-26.171}{28.367}=0.886
$$

La confiabilidad del instrumento de 40 ítems aplicado a los 1106 estudiantes, cuyas opciones fueron marcar sí o no, se calculó mediante el uso del Alfa de Cronbach. El objetivo de este instrumento fue verificar el criterio de 
los alumnos acerca del desarrollo de la competencia para el diseño y la ejecución del proceso de enseñanzaaprendizaje en sus profesores, antes y después de la capacitación. El resultado se alcanzó aplicando la fórmula (2), el 0.741 que resultó del cálculo también evidencia que el instrumento es confiable.

$$
a=\frac{k}{k-1}\left[1-\frac{\sum v i}{v t}\right]=\frac{40}{40-1} * \frac{\sum 5.7}{19.21}=0.741
$$

Se utilizó el análisis documental para estudiar planes de clases, evaluaciones docentes, actividades metodológicas y el sistema de superación. El método estadístico se puso en práctica para procesar la información recopilada en las entrevistas, la encuesta y la observación, establecer los resultados del diagnóstico y llegar a la caracterización del colectivo. El análisis y la síntesis se emplearon para procesar, seleccionar y redactar toda la información obtenida. El método genético para estudiar la evolución de la competencia para el diseño y la ejecución del proceso enseñanza-aprendizaje de los profesores de la FACAE. El método lógico para determinar y relacionar los componentes del sistema. Se introdujo el método sistémico para establecer la relación dialéctica y de dependencia entre los elementos que componen el sistema de capacitación y entre los ítems del instrumento creado para evaluar la competencia para el diseño y la ejecución del proceso enseñanzaaprendizaje, con el propósito de destacar que el comportamiento de uno de ellos influye en la manifestación del resto. También se usó el método hermenéutico en el proceso de interpretación de documentos, como antecedente de la redacción de aquellos en los que expresan los resultados de la investigación.

\section{RESULTADOS}

Los resultados se relacionan con la fundamentación de la propuesta y de las competencias en varias de sus dimensiones hasta llegar a la del diseño y la ejecución del proceso de enseñanza-aprendizaje. Se refieren, además, a los componentes del sistema de capacitación y sus logros. Se concretan en la sistematización de referentes teóricos y metodológicos relacionados con la mencionada competencia, en la elaboración y puesta en práctica del sistema de capacitación acerca de esta, en la creación de un instrumento con 40 indicadores para la evaluación de la misma. En una nueva definición de esta competencia y en la trasformación del modo de actuación de los docentes de la FACAE relacionado con la competencia y en la elevación, por consiguiente, de la satisfacción de los estudiantes con las clases que reciben, lo cual se refleja en el aprendizaje desarrollador y en la formación integral.

\section{Fundamentación de la propuesta}

Travé y Pozuelos (2017) destacan la importancia de formar maestros capaces de diseñar e implementar materiales creativos para mejorar la práctica en el aula y por ende el aprendizaje de sus alumnos. Sin embargo, Vilkancienè y Rozgienè (2017) señalan que los maestros aún carecen de competencias para la preparación y el diseño de materiales didácticos para sus clases. Proyectos que involucran a maestros, estudiantes, individuos, grupos de personas, tecnologías e instituciones, son más cotidianos y muestran que las condiciones para el proceso de enseñanza-aprendizaje se transforman continuamente. No obstante, también se observa que las tradiciones educativas ofrecen resistencia al cambio. Esto ha provocado una preocupación por la formación de profesores competentes, aptos para conducir acertadamente el aprendizaje de los estudiantes (Lind et al., 2014).

La formación del personal docente no se limita al pregrado. Las instituciones educativas deben estar atentas a los cambios que precisan modificaciones en el modo de actuación de los profesores. A tono con esos cambios estos profesionales deben someterse a un sistema de formación continua, durante la vida, que tiene un baluarte significativo en las capacitaciones. En a FACAE de la UTN se realizó una propuesta de capacitación docente sobre la base de un diagnostico relacionado con la competencia para el diseño y la ejecución del proceso de enseñanza- aprendizaje. El sistema capacitación propuesto es resultado de un proyecto de investigación que permitió conocer que el $86.29 \%$ de los docentes de la FACAE presenta deficiencias relacionadas con el desarrollo de esta competencia y que ello incide en la formación de los estudiantes.

La observación del modo de actuación profesional de los docentes posibilitó determinar las siguientes deficiencias. Existe un alto nivel de improvisación en el desarrollo de la clase, resultado de una insuficiente preparación previa y del sobredimensionamiento de la experiencia empresarial. Las categorías de la Didáctica no se aplican al diseño y a la ejecución de la clase, lo cual es resultado del desconocimiento del sistema teórico y práctico de esta ciencia. Prevalece el método expositivo, sustentado en el uso exagerado de diapositivas, al margen de la metodología para su correcta elaboración y utilización. El uso indiscriminado de este método no favorece la retroalimentación, el debate, la polémica ni el diálogo. Hubo otras deficiencias detectadas como las que se siguen. No se pone en práctica la estructura dialéctica de la secuencia armónica del desarrollo de la clase. Las actividades autónomas carecen de la base orientadora para la acción y son dogmáticas y memorísticas y el control que se realiza de ellas también lo es. La evaluación tiene carácter cuantitativo, no cumple con sus funciones, ni coloca al estudiante en posición de éxito. Las formas organizativas empleadas a 
veces no se corresponden con las características del contenido y de los alumnos. Los estudiantes expresaron una satisfacción baja con las clases que reciben.

Se demostró que las causas de estas deficiencias se relacionan con carencias en la formación de los docentes. Ecuador forma a sus profesores universitarios a corto y mediano plazos, mediante capacitaciones, cursos, conferencias, seminarios y talleres. Varios educadores de la FACAE son seleccionados por su condición de magísteres o doctores en ciencias $(\mathrm{PhD})$. Este procedimiento de selección posibilita el ingreso de algunos sin conocimientos especializados para el ejercicio de la docencia, lo cual incide en la formación de los estudiantes, especialmente en el desarrollo del aprendizaje. La experiencia laboral empresarial de estos profesionales y los títulos de cuarto nivel son potencialidades. Sin embargo, no haber formado a los profesores en el pregrado para su misión cardinal obliga a hacerlo después en ejercicio, por tanto, la capacitación docente es recurso que los coloca en condiciones de cumplir con esta responsabilidad de manera más eficiente.

\section{El sistema de capacitación}

Dentro del sistema de capacitación se concibieron 5 componentes esenciales, tales como la fundamentación teórica y metodológica de la competencia para el diseño y la ejecución del proceso de enseñanza-aprendizaje. El desarrollo de un curso de capacitación acerca de la misma. La elaboración, puesta en práctica y validación del instrumento de 40 ítems para la evaluación de esta competencia que sirve como guía para su desarrollo, y en una nueva definición de esta, la clase abierta como actividad metodológica demostrativa y el taller integrador, colofón de todo el sistema. El sistema se concentró en el dominio de la teoría de la Pedagogía y de la Didáctica como ciencias; en la diferenciación del objeto de estudio de cada una, de sus funciones y de la relación dialéctica entre ellas (Ramos et al., 2017). En la estructura de la clase y sus fases, en el orden de sus componentes, en la armonía entre ellos y en la utilidad de las categorías de la Didáctica, necesarias para su puesta en práctica (Abreu et al., 2018). Tuvo como núcleo, además, la importancia del método para organizar y dinamizar la clase en función de actividades que garanticen un aprendizaje significativo y desarrollador para los alumnos, que facilite el tránsito de la dependencia a la independencia y que sea duradero.

Otro aspecto priorizado fue la definición de los tipos de clases más apropiados, teniendo en cuenta las características del contenido de las materias, las del colectivo docente, los estudiantes y la organización del proceso de enseñanza-aprendizaje. Los más utilizados fueron la conferencia o clase magistral, concebida para la orientación general de los temas del sistema del contenido de las asignaturas. La clase práctica para el trabajo con las fuentes del contenido bajo la dirección del profesor. El seminario para profundizar en el dominio del contenido, después del estudio autónomo. Los talleres para la integración del contenido, el perfeccionamiento, enriquecimiento y creación del conocimiento nuevo, superior, integral y contextualizado. (Abreu et al., 2017).

Rios y Herrera (2017) hablan del rol formativo de la evaluación por competencias en el ámbito educativo, de su relación con los perfiles curriculares orientados a los nuevos contextos laborales. También analizan la necesidad de establecer lineamientos, diseños, implementaciones y desarrollos instrumentales para determinar los impactos de los aprendizajes por competencias, como una forma de evidenciar la apropiación de saberes integrales, múltiples y complejos. Gadušová et al., (2019) aseguran que las competencias docentes garantizan un nivel alto y sostenible de la educación. Proponen un modelo de evaluación de estos basado en 10 competencias. En tal sentido se preguntan si el profesor evalúa el progreso y los resultados del proceso de enseñanza y aprendizaje, utiliza los recursos materiales y las ayudas en el proceso de enseñanza, selecciona e implementa formas organizativas y métodos de enseñanza, planifica y diseña la enseñanza, domina y controla el contenido y la didáctica de las asignaturas que imparte, planifica y emprende su desarrollo profesional propio, crea un clima positivo en la clase, desarrolla la personalidad del alumno y sus competencias, identifica los factores psicológicos y sociales que intervienen en el aprendizaje de sus estudiantes y relaciona las características individuales de los alumnos con el desarrollo social.

Este modelo de evaluación de competencias docente está centrado en el proceso de enseñanza-aprendizaje, pero separa la enseñanza del aprendizaje, no lo concibe como un todo integrado armónico y holístico. No abarca todas las funciones del docente como agente personalizado de dicho proceso y no tiene a la clase como núcleo de su demostración. El sistema de capacitación incluyó un curso de capacitación cuya duración fue de 40 horas. Su objetivo fue capacitar a los docentes FACAE de la UTN en lo relacionado con el contenido y la metodología para el desarrollo de la competencia para el diseño y la ejecución del proceso de enseñanzaaprendizaje. Otras actividades que se incorporaron al sistema fueron el desarrollo de clases demostrativas abiertas en equipos de trabajo, para cuya observación y debate se utilizó como guía el instrumento creado por los investigadores. Por último, se realizó un taller integrador para debatir los aspectos que integran la competencia, especialmente aquellos en los que detectaron las mayores dificultades. La Tabla 1 muestra el sistema de capacitación con sus componentes, relaciona el tema, los objetivos, el tiempo de duración, los contenidos y las observaciones para su realización. 
Tabla 1. Componentes del curso de capacitación.

\begin{tabular}{|c|c|c|c|c|}
\hline Tema & Objetivo & $\begin{array}{l}\text { Tiempo de } \\
\text { duración }\end{array}$ & Contenidos & Observaciones \\
\hline $\begin{array}{l}\text { Las competencias } \\
\text { profesionales }\end{array}$ & $\begin{array}{l}\text { Explicar las } \\
\text { competencias } \\
\text { profesionales }\end{array}$ & 5 horas & $\begin{array}{l}\text { Los referentes teóricos y metodológicos de } \\
\text { las competencias profesionales. } \\
\text { Los tipos de competencias profesionales. } \\
\text { La importancia de las competencias } \\
\text { profesionales para la docencia. } \\
\text { El profesional competente para la docencia. }\end{array}$ & $\begin{array}{l}\text { Para el desarrollo de este tema se utilizó la } \\
\text { clase práctica como forma organizativa. El } \\
\text { trabajo consistió en el estudio de fuentes del } \\
\text { contenido. } \\
\text { Equipos de trabajo de dos integrantes } \\
\text { escribieron un ensayo o una ponencia y } \\
\text { evidenciaron los antecedentes del tema. } \\
\text { Finalmente se desarrolló un seminario en el } \\
\text { que se presentaron y debatieron los trabajos. }\end{array}$ \\
\hline $\begin{array}{l}\text { Las } \\
\text { competencias } \\
\text { del profesional } \\
\text { de la educación }\end{array}$ & $\begin{array}{l}\text { Caracterizar } \\
\text { las } \\
\text { competencias } \\
\text { profesionales } \\
\text { para la } \\
\text { docencia }\end{array}$ & 10 horas & $\begin{array}{l}\text { Competencia cognoscitiva. } \\
\text { Competencia para el diseño y la ejecución } \\
\text { del proceso de enseñanza-aprendizaje. } \\
\text { Competencia comunicativa orientadora. } \\
\text { Competencia investigativa. }\end{array}$ & $\begin{array}{l}\text { Se utilizó la conferencia como forma } \\
\text { organizativa. } \\
\text { Se realizaron presentaciones y ejercicios } \\
\text { prácticos sustentados en la problematización } \\
\text { del proceso de enseñanza-aprendizaje, para } \\
\text { lograr el objetivo. }\end{array}$ \\
\hline $\begin{array}{l}\text { La competencia } \\
\text { para el diseño del } \\
\text { y la ejecución } \\
\text { proceso de } \\
\text { enseñanza- } \\
\text { aprendizaje }\end{array}$ & $\begin{array}{l}\text { Demostrar la } \\
\text { competencia } \\
\text { para } \\
\text { el diseño y la } \\
\text { ejecución del } \\
\text { proceso de } \\
\text { enseñanza- } \\
\text { aprendizaje. }\end{array}$ & 20 horas & $\begin{array}{l}\text { Los referentes teóricos y metodológicos de } \\
\text { la competencia para del proceso de } \\
\text { enseñanza-aprendizaje. } \\
\text { La definición de la competencia para el } \\
\text { diseño y la ejecución del proceso de } \\
\text { enseñanza-aprendizaje. } \\
\text { Las habilidades didácticas para el diseño } \\
\text { del proceso de enseñanza-aprendizaje. } \\
\text { La motivación por la actividad de diseño y } \\
\text { la ejecución del proceso de enseñanza- } \\
\text { aprendizaje. } \\
\text { Las características actitudinales asociadas } \\
\text { con la competencia para el diseño y la } \\
\text { ejecución del proceso de enseñanza- } \\
\text { aprendizaje. } \\
\text { La capacidad metodológica asociada con la } \\
\text { competencia para el diseño y la ejecución } \\
\text { del proceso de enseñanza-aprendizaje. } \\
\text { Las cualidades integradoras que revelan el } \\
\text { desarrollo de la competencia para el diseño } \\
\text { y la ejecución del proceso y la ejecución } \\
\text { de enseñanza-aprendizaje. }\end{array}$ & $\begin{array}{l}\text { En este tema se integraron varias formas } \\
\text { organizativas. La conferencia, la clase } \\
\text { práctica, la actividad autónoma y el taller } \\
\text { integrador. La primera tuvo un carácter } \\
\text { panorámico y orientador. La segunda se } \\
\text { dirigió al trabajo con documentos y la } \\
\text { preparación de clases que evidenciaran el } \\
\text { desarrollo de la competencia. } \\
\text { Las actividades del trabajo autónomo se } \\
\text { orientaron a la búsqueda, indagación y } \\
\text { constatación de información relacionada con } \\
\text { el contenido de cada temática. Tuvieron el } \\
\text { propósito de desarrollar habilidades que } \\
\text { garantizaron la profundización del dominio } \\
\text { del contenido y la independencia } \\
\text { cognoscitiva. } \\
\text { Los talleres propiciaron la integración del } \\
\text { contenido del tema. Su finalidad fue el } \\
\text { debate profundo y reflexivo del mismo y la } \\
\text { aplicación a situaciones relacionadas con el } \\
\text { diseño y la ejecución del proceso de } \\
\text { enseñanza-aprendizaje de las materias de } \\
\text { cada profesor. }\end{array}$ \\
\hline
\end{tabular}

\section{Indicadores}

Son la base para que un evaluador juzgue si un profesional es o no competente. Sustentan la elaboración del material de evaluación. Permiten precisiones acerca de lo que se hizo y la calidad con que fue realizado. Se elaboraron 40 indicadores de la competencia para el diseño y la ejecución del proceso de enseñanzaaprendizaje, los cuales se muestran en la Tabla 2, y se estableció una escala de valores ascendentes del 1 al 5 para la evaluación de los mismos, en la que se considera el 1 como la evaluación mínima y el 5 como máxima.

\section{Las clases demostrativas abiertas}

Una vez concluida la capacitación de los docentes de la FACAE se organizó este tipo de actividad metodológica. Se crearon equipos de trabajo por carreras, cuyo número de integrantes osciló entre 4 y 5 , según la cantidad de profesores de estas. Este tipo de actividad docente-metodológica constituyó un acto académico significativo del sistema de capacitación. En cada equipo se seleccionó por azar 1 docente para que impartiera una clase abierta que fue preparada en colectivo. La clase se impartió con la presencia de todos los integrantes de cada equipo, los cuales contaron con la guía de observación en la que se incluyeron los indicadores relacionados con la competencia para el diseño y la ejecución del proceso de enseñanzaaprendizaje elaborados por los investigadores. En total se desarrollaron 21 clases. La Figura 1 muestra los resultados de la evaluación de los indicadores durante el desarrollo de las clases demostrativas abiertas. 
Tabla 2. Indicadores para la evaluación de la competencia para el diseño y la ejecución del proceso de enseñanzaaprendizaje.

\begin{tabular}{|c|c|c|c|c|c|c|}
\hline \multirow[t]{3}{*}{$\begin{array}{l}\text { Fases de la } \\
\text { clase }\end{array}$} & \multirow{3}{*}{\multicolumn{2}{|c|}{ Indicadores }} & \multicolumn{4}{|c|}{$\begin{array}{l}\text { Escala de } \\
\text { valores }\end{array}$} \\
\hline & & & \multicolumn{4}{|r|}{$(+)$} \\
\hline & & & 1 & 2 & 3 & \begin{tabular}{l|l}
44 & 5
\end{tabular} \\
\hline $\begin{array}{l}\text { Preparación } \\
\text { previa }\end{array}$ & & $\begin{array}{l}\text { Dominio del contenido de la materia que imparte en función del diagnóstico, el pronóstico, la selección y } \\
\text { la estructuración del mismo. } \\
\text { Dominio de las fuentes para el estudio del contenido de la materia que imparte. } \\
\text { Dominio de la estructura curricular de la materia que imparte. } \\
\text { Dominio del modelo pedagógico de la institución donde labora. } \\
\text { Dominio del modelo didáctico de la institución donde labora. } \\
\text { Conocimientos acerca de la caracterización de sus estudiantes. } \\
\text { Conocimientos acerca de la Pedagogía, la Didáctica y la metodología para concebir la clase. } \\
\text { Conocimientos acerca de actividades extradocentes, extraescolares y autónomas. } \\
\text { Conocimientos acerca desarrollo de las habilidades de diseño y vías para autopotenciarlas, } \\
\text { autorregularlas, autoperfeccionarlas y autoevaluarlas. } \\
\text { Conocimientos acerca de organización del proceso de enseñanza-aprendizaje. }\end{array}$ & & & & \\
\hline Introducción & & $\begin{array}{l}\text { Crea condiciones organizativas para el desarrollo de la clase. } \\
\text { Controla y evalúa el trabajo autónomo de forma tal que contribuye a la presentación del nuevo tema. } \\
\text { Presenta el tema de la clase. } \\
\text { Motiva a los estudiantes para la clase. } \\
\text { Evidencia haber dedicado tiempo, energía y esfuerzo intelectual a la preparación previa. } \\
\text { Deriva, determina y formula objetivos formativos contextualizados y relacionados con aspiraciones } \\
\text { individuales y sociales. } \\
\text { Enuncia el objetivo de la clase. }\end{array}$ & & & & \\
\hline Desarrollo & & $\begin{array}{l}\text { Domina profundamente el contenido del tema de la clase. } \\
\text { Demuestra haber preparado y concebido la clase para todos los alumnos, sobre la base del diagnóstico } \\
\text { y de la diversidad del colectivo estudiantil. } \\
\text { Selecciona, establece la secuencia y organiza el contenido de la clase según las características de los } \\
\text { estudiantes y del contexto. } \\
\text { Aplica formas organizativas adecuadas a las características del colectivo estudiantil y a las exigencias } \\
\text { del contenido de la clase. Utiliza métodos, medios y procedimientos que garantizan la participación } \\
\text { activa de todos los estudiantes y aseguran el aprendizaje de estos, sin discriminación, ni favoritismo. } \\
\text { Pone en práctica la evaluación cualitativa, convenida con los estudiantes, con todas sus funciones, en } \\
\text { diversidad de formas, no coercitiva, no para sancionar el error; sino para estimular y colocar al estudiante } \\
\text { en posición de éxito. } \\
\text { Emplea las TIC y estimula a los estudiantes para que lo hagan. } \\
\text { Planifica, orienta y controla el trabajo autónomo de los estudiantes atendiendo a las diferencias } \\
\text { individuales. } \\
\text { Utiliza fuentes del contenido variadas, diversas, novedosas y actualizadas y las orienta resaltando } \\
\text { debilidades y potencialidades para el estudio del contenido e indica el lugar de localización para su } \\
\text { consulta. } \\
\text { Demuestra conocimientos acerca de la Pedagogía, la Didáctica y la metodología para desarrollar la } \\
\text { clase. } \\
\text { Desarrolla habilidades. } \\
\text { Forma y desarrolla valores. } \\
\text { Conduce correctamente toda la clase. } \\
\text { Estimula el debate, la discusión, la polémica, el dialogo y el trabajo en equipos. } \\
\text { Logra una comunicación asertiva entre todos los participantes. } \\
\text { Vincula la clase con la investigación. } \\
\text { Propicia el aprendizaje significativo y desarrollador de los estudiantes. } \\
\text { Logra que sus estudiantes manifiesten satisfacción con el desarrollo de la clase. } \\
\text { Mantiene la motivación de los estudiantes durante su desarrollo. }\end{array}$ & & & & \\
\hline Conclusiones & & $\begin{array}{l}\text { Orienta el trabajo autónomo de manera tal que el control que realice del mismo sirva para establecer el } \\
\text { vínculo entre la última clase y la siguiente. } \\
\text { Comprueba si se logró el objetivo de la clase. } \\
\text { Realiza las conclusiones de la clase y puntualiza los aspectos más destacados. } \\
\text { Vincula el tema estudiado con el que se tratará en la próxima clase. }\end{array}$ & & & & \\
\hline
\end{tabular}




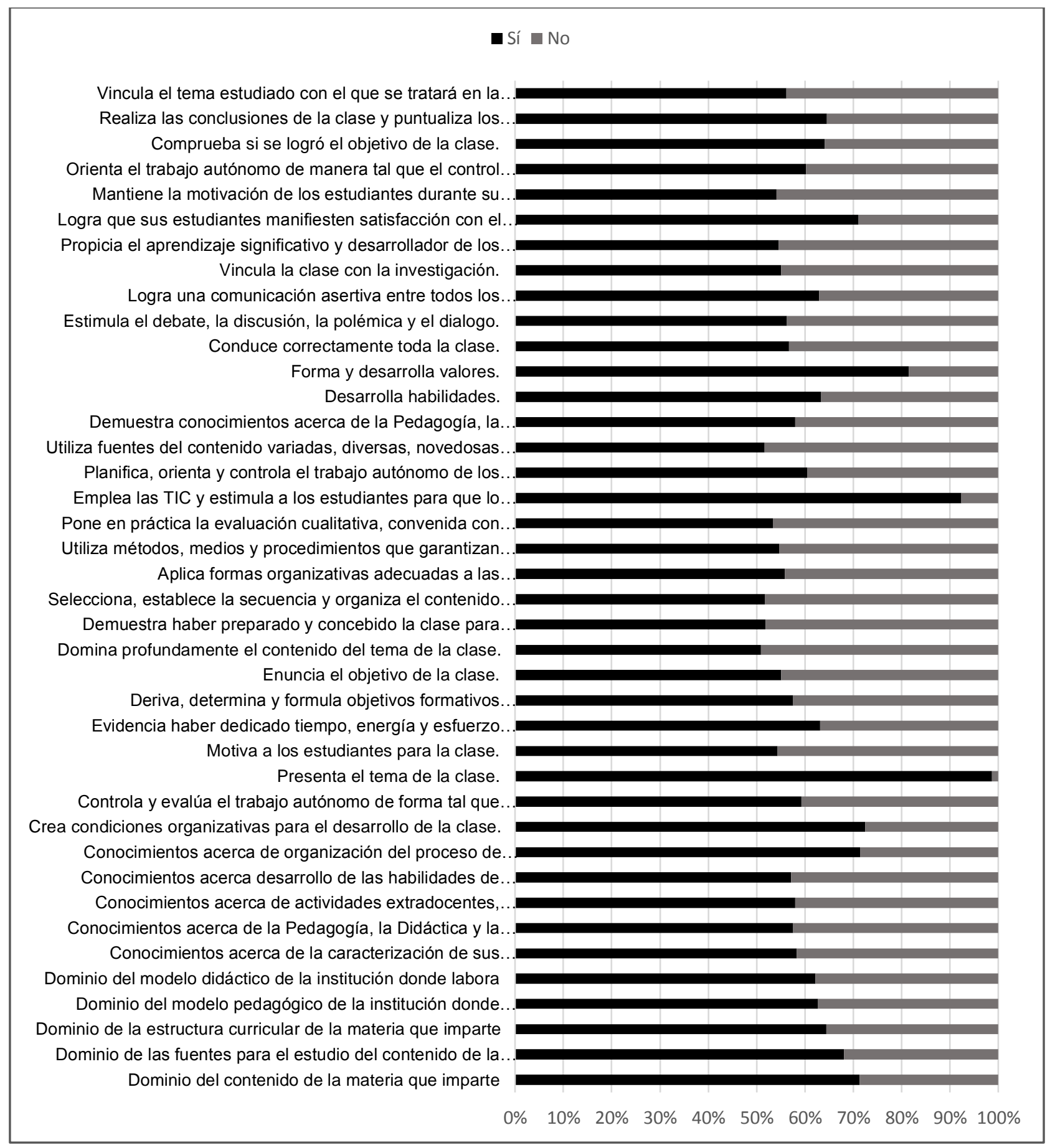

Fig. 1. Comportamiento de los indicadores de la competencia para el diseño y la ejecución del proceso de enseñanzaaprendizaje después del sistema de capacitación.

Como se aprecia en la Figura 1 todos los indicadores alcanzaron calificaciones por encima del $50 \%$ de positividad, superando las del diagnóstico donde oscilaron entre 32 y $61 \%$. Los de mejores resultados fueron: emplea las TIC y estimula a los estudiantes para que lo hagan, forma y desarrolla valores en la clase. Otros como crea condiciones organizativas para el desarrollo de la clase, y presenta el tema, también recibieron evaluación positiva. Los resultados más bajos fueron para los indicadores propicia el aprendizaje significativo y desarrollador de los estudiantes, que aunque fue superior al diagnóstico aun no alcanza los niveles deseados; pone en práctica la evaluación cualitativa, convenida con los estudiantes, con todas sus funciones, en diversidad de formas, no coercitiva, no para sancionar el error; sino para estimular y colocar al estudiante en posición de éxito y motiva a los estudiantes para la clase, en orden descendente. El indicador logra que sus estudiantes manifiesten satisfacción con el desarrollo de la clase, aumentó de un $65 \%$, antes de la capacitación a un $71 \%$. Finalmente, se desarrolló un taller integrador que permitió centrar la atención en los indicadores de más bajas calificaciones. Se realizaron actividades demostrativas relacionadas con estos. Se aprovecharon las potencialidades evidenciadas para reforzar los conocimientos y se hizo la evaluación final, cuyos resultados fueron favorables. 


\section{DISCUSIÓN}

El ejercicio de la docencia es tan necesario como complejo. Las sociedades ocupadas en el mejoramiento de la calidad de vida de sus habitantes se interesan en el perfeccionamiento de sus sistemas educativos. El recurso humano es esencial para hacer realidad esta aspiración universal. Contar con docentes competentes es una cuestión de primer orden para el mundo de hoy. Los estudios relacionados con las competencias docentes son más numerosos, diversos y valiosos, en la medida en que en las naciones se comprende que la educación es un componente básico del desarrollo. La educación superior tiene el objetivo de preparar a los estudiantes para que ingresen en el mercado laboral exitosamente. El proceso de enseñanza-aprendizaje y su entorno cambian contantemente. Este escenario dinámico necesita de profesores competentes, capaces de diseñar un proceso en el que sus agentes personalizados interactúen en función de un aprendizaje efectivo (Geitz et al., 2019).

\section{Competencias para el proceso de enseñanza-aprendizaje}

Soares de Vasconcelos y de Armas (2016) plantean que en el proceso de enseñanza- aprendizaje es esencial saber no solo cómo aprenden los estudiantes, sino qué metodología emplean los maestros para lograr un aprendizaje efectivo. Zhu et al., (2013) afirman que existe una relación dialéctica entre esta competencia y el desarrollo del proceso de enseñanza-aprendizaje innovador. El docente debe desarrollar competencias, tener una formación en contenidos científicos, didácticos y metodológicos y realizar proyectos innovadores. Tejada (2009) afirma que existe una competencia de carácter general que le permite a este planificar, impartir, tutorar y evaluar acciones formativas, elaborar y utilizar medios y recursos didácticos que estimulen la calidad de la formación y la actualización didáctica.

En opinión de Moreno et al., (2015) esta competencia está integrada por diferentes unidades que consisten en programar acciones formativas, proporcionar oportunidades de aprendizaje, verificar el nivel de adquisición de las competencias profesionales, los programas y las acciones y contribuir activamente a la mejora de la calidad de la formación. Cada unidad cuenta con sus realizaciones propias y tiene como objetivo proporcionar un aprendizaje con significado y funcionalidad. Consiste, además, en poner en práctica un conjunto de conocimientos, actitudes, habilidades y valores duraderos en el desarrollo de la actividad profesional. Por último, involucra al estudiante de manera activa, fomenta la reflexión, estimula el pensamiento crítico y permite tomar decisiones, resolver problemas, diseñar proyectos.

Según Pla (2005 la competencia para el diseño y la ejecución del proceso de enseñanza-aprendizaje consiste en la configuración psicológica de la personalidad. Es el constructo que designa la idoneidad del docente para planificar de forma creadora y contextualizada el proceso educativo y realizar la transposición didáctica del contenido de la cultura universal a la actividad de educación del alumno. En ella se destacan las acciones específicas dirigidas a diagnosticar, pronosticar, seleccionar y estructurar contenidos, seleccionar fuentes, determinar los métodos, procedimientos, tareas docentes, concebir la evaluación y las formas de organización, para los diferentes tipos de diseño y se manifiesta en el desempeño del profesor mediante la capacidad metodológica. Hernández et al., (2015) destacan la necesidad del desarrollo de esta competencia en los docentes. Plantean que en el proceso de enseñanza-aprendizaje, cuya dinámica se expresa en la relación docente estudiante, es de vital importancia tener en cuenta las competencias que los docentes deben desarrollar. Aseguran que esta es la manera de garantizar la calidad del desempeño profesional de los profesores en función de la formación integral de sus discípulos.

\section{Competencias en las fases de la clase}

Como se expresó anteriormente la competencia para el diseño y la ejecución del proceso de enseñanzaaprendizaje está estrechamente asociada a la clase y se manifiesta en cada una de sus fases; la preparación previa, la introducción, el desarrollo y las conclusiones. La clase es un proceso académico de elevada complejidad organizado institucionalmente, en el que intervienen dos agentes personalizados, el educador y los educandos. Es el eslabón básico y esencial del proceso de enseñanza-aprendizaje desarrollador. En ella se establecen relaciones interpersonales dialécticas y dinámicas que deben sustentarse en el análisis, el dialogo, el debate, la reflexión, el respeto, la comprensión, la tolerancia, la camaradería, el trabajo en equipo y la exigencia. Tiene como núcleo un sistema de contenidos que se nutre de la cultura universal, se expresa mediante información diversa y novedosa revelada en esquemas, modelos, teorías, paradigmas, hechos, conceptos, enfoques, regularidades y leyes relacionados entre sí, que integra conocimientos, habilidades, normas de conducta, maneras de pensar, sentimientos y valores, que se selecciona en dependencia de sus potencialidades educativas para la formación integral de un ser humano, sobre la base de la conjugación de intereses individuales, grupales, colectivos y sociales y se estudia, asimila y enriquece mediante la práctica de objetivos, métodos, medios, técnicas, procedimientos, actividades, fuentes diversas y la evaluación cualitativa. 


\section{La preparación previa de la clase}

Está competencia se habrá logrado si en la preparación previa de la clase el docente demuestra que domina el contenido de la materia que imparte, en función del diagnóstico, el pronóstico, la selección y la estructuración del mismo. Si domina, además, las fuentes para el estudio del mismo, su estructura curricular y el modelo pedagógico y didáctico de la institución donde labora. Si evidencia que conoce la caracterización de sus estudiantes, la Pedagogía, la Didáctica y la metodología para concebir la clase, las actividades extradocentes, extraescolares y autónomas que debe preparar, el desarrollo de las habilidades para el diseño y vías para autopotenciarlas, autorregularlas, autoperfeccionarlas y autoevaluarlas, y la organización del proceso de enseñanza-aprendizaje.

\section{La introducción de la clase}

En la introducción de la clase el docente debe crear condiciones organizativas para el desarrollo de esta, controlar y evalúa el trabajo autónomo orientado en la clase anterior, de forma tal que contribuye a la presentación del nuevo tema. También deberá, motivar a los estudiantes, evidenciar haber dedicado tiempo, energía y esfuerzo intelectual a la preparación previa, y derivar, determinar, formular y enunciar los objetivos formativos contextualizados y relacionados con aspiraciones individuales y sociales.

\section{El desarrollo de la clase}

Durante el desarrollo de la clase el docente debe demostrar que domina profundamente el contenido del tema, que preparó y concibió la clase para todos los alumnos, sobre la base del diagnóstico y la diversidad del colectivo estudiantil, que estableció la secuencia y organizó el contenido según las características de los estudiantes y del contexto, que aplica formas organizativas adecuadas a las características del colectivo estudiantil y a las exigencias del contenido, que utiliza métodos, medios y procedimientos que garantizan la participación activa de todos los estudiantes y aseguran el aprendizaje desarrollador de estos, sin discriminación, ni favoritismo. Además, que pone en práctica la evaluación cualitativa, convenida con los estudiantes, con todas sus funciones, en diversidad de formas, no coercitiva, no para sancionar el error; sino para estimular y colocar al estudiante en posición de éxito. En esta fase el profesor debe demostrar adicionalmente que emplea las TIC y estimula a sus alumnos para que lo hagan, que planifica, orienta y controla el trabajo autónomo atendiendo a las diferencias individuales, que utiliza fuentes del contenido variadas, diversas, novedosas, actualizadas y las orienta resaltando debilidades y potencialidades para el estudio del contenido e indica el lugar de localización para su consulta. Asimismo, que conoce de Pedagogía, Didáctica y metodología para el progreso de la clase, la conduce correctamente, forma y desarrolla valores y habilidades, estimula el debate, la discusión, la polémica, el dialogo y el trabajo en equipos y que logra una comunicación asertiva entre todos los participantes. Por último, que vincula a la clase con la investigación, propicia el aprendizaje significativo y desarrollador, mantiene la motivación y logra que sus discípulos manifiesten satisfacción con el desarrollo de la clase

\section{Las conclusiones de la clase}

Finalmente, en las conclusiones de la clase orienta el trabajo autónomo de manera tal que el control que realice del mismo sirva para establecer el vínculo entre la última clase y la siguiente, comprueba si se logró el objetivo, realiza las conclusiones, puntualiza los aspectos más destacados y vincula el tema estudiado con el que se tratará en la próxima clase.

\section{CONCLUSIONES}

La competencia para el diseño y la ejecución del proceso de enseñanza-aprendizaje está asociada directamente a las fases más importantes de la clase, la preparación previa, la introducción, el desarrollo y las conclusiones. Sobre la base de un diagnostico que demostró su necesidad, se implementó un sistema capacitación, mediante el cual se logró el desarrollo de esta competencia en los docentes de la FACAE de la UTN. El instrumento elaborado para evaluar esta competencia fue un factor influyente en el desarrollo de la misma. Este resultado elevó la satisfacción de los estudiantes por la calidad de las clases y favoreció el aprendizaje significativo y desarrollador de estos.

\section{NOTACIÓN}

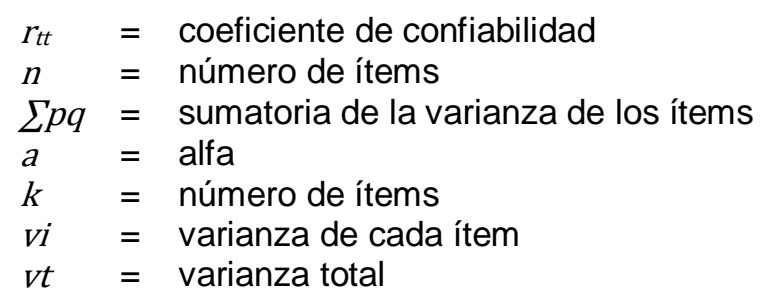




\section{REFERENCIAS}

Abreu, O., Rhea, S., Arciniegas, G., y Rosero, M., Objeto de estudio de la didáctica: análisis histórico epistemológico y crítico del concepto. doi.org/10.4067/S0718-50062018000600075, Formación Universitaria11, (6), 75-82 (2018)

Abreu, O., Gallegos, M., Jácome, J., y Martínez, R., La didáctica: epistemología y definición en la Facultad de Ciencias Administrativas y Económicas. doi.org/10.4067/S0718-50062017000300009, Formación Universitaria, 10 (3) 81-92 (2017)

Abreu, O., M. Naranjo, S. Rhea y Gallegos, M., Modelo didáctico para la Facultad de Ciencias Administrativas y Económicas de la Universidad Técnica del Norte en Ecuador. doi.org/10.4067/S0718-50062016000400002, Formación Universitaria, 9 (4) 3-10 (2016)

Barreto da Cruz, G. y Andrade, P., The teaching of didactics and the teacher trainer's role from the perspective of Visual Arts teaching students. doi.org/10.1590/s1517-9702201701156893, Educ. Pesqui, 43 (2) 483-498 (2017)

Caena, F. y Redecker, C., Aligning teacher competence frameworks to $21^{\text {st }}$ century challenges: The case for the European Digital Competence Framework for Educators. doi.org/10.1111/ejed.12345, European Journal of Education, 54, 356-369 (2019)

Gadušová Z., Jakubovská, V., Markechová, D., y Tirpáková, N., Teacher Competences Development - a Guarantee of Sustainable High Level of Education and Training. doi.org/10.18421/TEM83-52, TEM Journal, 8 (3), 1063-1070(2019)

Geitz, G., De Geus, J., y Tinoca, J., Designbased education, sustainable teaching, and learning. doi.org/10.1080/2331186X.2019.1647919, Cogent Education, 6:1, 1647919, 1-15 (2019)

Gokalp, M., Investigating classroom teaching competencies of pre service elementary mathematics teachers. Eurasia Journal of Mathematics, Science and Technology Education. ISSN: 1305-8223, 12 (3), 503-512 (2016)

Guasch, T., Alvarez, I., y Espasa, A., University teacher competencies in a virtual teaching/learning environment: Analysis of a teacher training experience. doi.org:10.1016/j.tate.2009.02.018, Teaching and Teacher Education., 26, 199-206 (2010)

Guerrero, D. y De los Ríos, I., Professional competences: a classification of international models, Procedia - Social and Behavioral Sciences, ISSN:1877- 0428, (46), 1290 - 1296 (2012)

Hernández, I., Recalde, J. y Luna, J. A., Estrategia didáctica: una competencia docente en la formación para el mundo laboral, Revista Latinoamericana de Estudios Educativos. ISSN: 1900-9895, 11 (1), 73-94 (2015)

Lund, A., Furberg, A., Bakken, J. y Engelien, K. L., What Does Professional Digital Competence Mean in Teacher Education? Nordic Journal of Digital Literacy. ISSN: 1891-943X, 9 (4), 281-299 (2014)

Medina, E. y Tabón, S., Formación integral y competencias. Pensamiento complejo, currículo, didáctica y evaluación, Revista Interamericana, ISSN: 0188-8838, 32 (2), 90-95 (2010)

McCabe, A. y O'Connor, U., Student-centred learning: the role and responsibility of the lecturer doi.org/10.1080/13562517.2013.860111, Teaching in Higher Education, 19 (4), 350-359 (2014)

Mohamadi, Z. y Malekshahi, N., Designing and validating a potential formative evaluation inventory for teacher competences. doi.org/10.1186/s40468-018-0059-2, Lang Test Asia, 8 (6), 2-21 (2018)

Moreno, J. A., Silveira, Y. y Belando, N., Questionnaire evaluating teaching competencies in the university environment. Evaluation of teaching competencies in the university. doi.org/10.7821/naer.2015.1.106, New approaches in educational research., 4 (19), 54-61 (2015)

Nousiainen T., Kangas, M., Rikala J, y Vesisenaho, M., Teacher competencies in game-based pedagogy. doi.org/10.1016/j.tate.2018.04.012,Teaching and Teacher Education, 74, 85-97 (2018)

Pla, R., Las competencias profesionales para el desempeño del docente en la educación de los alumnos desde un enfoque integrador. Curso 51, Pedagogía (2005)

Ramos, J. M., Rhea, B. S., Pla, R.V. y Abreu, O., La pedagogía como ciencia para el tratamiento de los contenidos generales del proceso educativo y la formación de valores. doi.org/10.4067/S0718-50062017000600001, Revista Formación Universitaria, 10 (6), 77-85 (2017)

Ríos, D. y Herrera, D., The challenges of competence-based assessment in the educational field. doi.org/10.1590/s16784634201706164230, Educação e Pesquisa., 43 (4), 1073-1086 (2017)

Santos, C. y Sarceda, C., Desarrollo de competencias docentes en educación infantil. Una experiencia interdisciplinar en la formación inicial de profesores. doi.org/10.4067/S0718-50062017000600005, Formación Universitaria, 10 (6) (2017)

Selvi, K., Teachers' competencies, International journal of philosophy of culture and axiology. ISSN: 2065-5002, 2 (1), 167176 (2010)

Soares de Vasconcelos, L. y De Armas, R., Compared education study: curriculum design for the development of competences. doi.org: 10.18543/the, Tuning Journal for Higher Education, (2), 319-346 (2016)

Stefanova, E. y otros 5 autores., Participatory model for identifying and measuring teachers' competences for open and Inquiry-based learning in STEM: field experience. EPiC Series in Education Science., ISSN: 2516-2306, 2, 28-39 (2019)

Tejada, J., Competencias docentes. doi.org/ 10.30827/profesorado.v24i1.8237, Profesorado, Revista de Currículum y Formación de Profesorado., 13, (2), 1-15 (2009) 
Travé, G. y Pozuelos, F.J., How teachers design and implement instructional materials to improve classroom practice. doi.org/10.3926/ic, 1062, Intangible Capital., 13 (5), 967-1043 (2017)

Vargas, C. y Apablaza, H., Competencia comunicativa en la formación inicial actual del profesor de matemática en Chile. doi.org/10.4067/S0718-50062019000300081, Formación Universitaria., 12 (3), 81-90 (2019)

Vilkancienè, L. y Rozgienè, I., CLIL teacher competences and attitudes. doi.org/10.1515/sm-2017-0019, Sustainable multilingualism darnioji daugiakalbyste., 11 (1)196-218, (2017)

Villarroel, V. A. y Bruna, B. D., Competencias pedagógicas que caracterizan a un docente universitario de excelencia: un estudio de caso que incorpora la perspectiva de docentes y estudiantes. doi.org/10.4067/S0718-50062017000400008, Formación Universitaria., 10 (4), 75-96 (2016)

Zhu C., Wang, D., Cai, Y. y Engels, N., What core competencies are related to teachers' innovative teaching? doi.org/10.1080/1359866X.2012.753984, Asia-Pacific Journal of Teacher Education., 41 (1), 9-27 (2013) 